\title{
Iron and Ferritin Levels of Children and Adolescents with Attention Deficit Hyperactivity Disorder and Attention Deficit Hyperactivity Disorder-Not Otherwise Specified
}

\author{
(1) Yusuf Öztürk1, (1) Zehra Topal2, (1) Nuran Demir33, (1) Ali Evren Tufan4 \\ 1Bolu Abant izzet Baysal University Faculty of Medicine, Department of Child and Adolescent Psychiatry, Bolu, Turkey \\ ${ }^{2}$ Gaziantep University Faculty of Medicine, Department of Child and Adolescent Psychiatry, Gaziantep, Turkey \\ 3izzet Baysal Hospital for Maternity and Pediatrics, Clinic of Child and Adolescent Psychiatry, Bolu, Turkey \\ ${ }^{4}$ Acıbadem University Faculty of Medicine, Department of Child and Adolescent Psychiatry, İstanbul, Turkey
}

\begin{abstract}
Aim: The study aimed to compare the levels of iron and ferritin in children with Attention Deficit Hyperactivity Disorder (ADHD) and AttentionDeficit Hyperactivity Disorder-Not Otherwise Specified (ADHD-NOS) and to assess the relationship between ADHD symptom severity and anxiety symptom severity with iron and ferritin levels.

Materials and Methods: This study was planned as a cross-sectional, retrospective study. The study was performed by scanning the records of patients who applied to our clinic between January 2012 and January 2013. Accordingly, 205 ADHD and ADHD-NOS case records were evaluated. Patients were diagnosed clinically according to Diagnostic and Statistical Manual of Mental Disorders, $4^{\text {th }}$ Edition, Text Revision (DSM-IV-TR) criteria. ADHD symptom severity was assessed by the Turgay DSM-IV-TR-Based Child and Adolescent Behavior Disorders Screening and Rating scale. Anxiety symptom severity was assessed by The Screen for Anxiety Related Emotional Disorders.

Results: Among the whole sample, 99 (48.3\%) patients had ADHD and 106 (51.7\%) had ADHD-NOS. In the ADHD group, the average age of the children was $10.88 \pm 3.02$ years, while that of the children in the ADHD-NOS group was $9.93 \pm 2.49$ years. Iron and ferritin were measured in 81 of the 205 patients participating in the study. There was no statistically significant difference between the two groups in terms of iron or ferritin levels $(p>0.05)$. Statistically significant negative correlations between ADHD hyperactivity symptom severity and iron levels, and ADHD attention deficit symptom severity and ferritin levels were found. Ferritin levels correlated statistically with the total number of psychiatric diagnoses in the children.

Conclusion: Iron and ferritin levels may be differentially affected in children with ADHD. The results we obtained from our study should be supported by studies with larger samples.
\end{abstract}

Keywords: ADHD, iron, ferritin

\section{Introduction}

Attention Deficit Hyperactivity Disorder (ADHD) is defined as an important childhood neuropsychiatric disorder with different clinical features, such as inattention, hyperactivity, and impulsivity. It is also often related to cognitive deficit (1). It is reported that the prevalence of ADHD ranges from $8.0 \%$ to $12.0 \%$ worldwide (2). The prevalence of ADHD in school-age children was determined to be $8.1 \%$ in Turkey (3). Attention-Deficit Hyperactivity Disorder Not Otherwise Specified (ADHDNOS) is a diagnosis in Diagnostic and Statistical Manual of Mental Disorders, $4^{\text {th }}$ Edition, Text Revision (DSM-IV-TR) 
for disorders with pronounced symptoms of inattention/ hyperactivity-impulsivity that do not meet the ADHD criteria (4). Faraone et al. (5) showed that individuals with ADHD and ADHD-NOS had similar patterns of functional impairment, comorbid psychiatric disorders, and familial inheritance. ADHD-NOS, which has been replaced by the term ADHD- Unspecified in DSM-5, is a diagnosis that can be made for situations where children may experience moderate attention problems that may affect learning or executive function in school (4). In addition, rather than being categorized as ADHD Other Specified and Unspecified types, many young people are defined as having borderline or subclinical levels of ADHD instead. Pharmacotherapy including stimulants and atomoxetine is the first choice in the treatment of both these disorders (2).

There are many theories regarding the etiology of ADHD, but it is not yet fully understood. A clear identifiable factor has not been demonstrated, but ADHD is known to be multifactorial (6). Studies have reported that the hereditary ratio of ADHD varies between $76.0 \%$ and $80.0 \%$ $(7,8)$. The exact cause is still unknown, but various prenatal and perinatal factors such as socio-psychological stress, exposure to toxins and heavy metals, diet, deficiency of neurotransmitters, gene variants and structural/functional abnormalities of the brain have been reported to contribute to the etiology $(2,7)$. ADHD is also frequently accompanied by comorbid conditions including iron deficiency (ID), electroencephalographic abnormalities, epilepsy, learning disabilities and depressive disorders (9).

Iron has an important role in systems that have vital features for the organism, such as the immune system, oxygen transport to cells, deoxyribonucleic acid synthesis and nitric oxide metabolism (10). In addition, iron is required in neurochemistry, proper brain morphology, and bioenergy processes (11). It has been shown that poor brain myelination associated with ID has a long-term effect on behavior in the early development period $(12,13)$. The regions where brain iron concentrations are highest are the Globus pallidus, substantia nigra, red nucleus, nucleus caudate and putamen (14). Fast iron accumulation in these areas is essential for the development of the brain and can contribute significantly to behavioral organization (14). Iron is a co-factor of tyrosine hydroxylase, an enzyme important in dopamine synthesis. Dopamine plays a significant role in ADHD pathophysiology (15). Various studies trying to elucidate ADHD neurobiology have shown that nutritional factors can affect brain function and play a role in the pathogenesis of ADHD (16-18). ID, which is one of these nutritional factors, is important because it plays a crucial role in the organizing of dopaminergic activity associated with the pathogenesis and symptoms of ADHD (19). Sever et al. (20) showed that children who were given iron supplements had increased ferritin levels and had decreased ADHD symptoms. These results suggest that iron supplements may be beneficial in children with non-anemic ADHD. In a systematic review examining the relationship between iron and ADHD, statistical analysis on iron status indices has not been shown (21).

Studies have shown strong evidence that ID in young children gives rise to developmental delays (22). ID has also been found to be related to cognitive changes in adolescents (23). In one study on rats, behavioral changes were shown to continue despite the ID being treated (24). Children with ID have more anxiety and/or depression with attentional and social problems (25).

We aimed to compare the levels of iron, ferritin and hemoglobin in children with ADHD and ADHD-NOS in this study. We also aimed to assess the relationships of ADHD symptom severity and anxiety symptom severity with respect to iron, ferritin and hemoglobin levels.

\section{Materials and Methods}

\section{Study Center and Time-frame}

The study was planned as a cross-sectional, retrospective study. It was conducted at the outpatient department of the Bolu Abant Izzet Baysal University Faculty of Medicine, Department of Child and Adolescent Psychiatry. The study was performed by scanning the records of patients who applied to our clinic between January 2012 and January 2013. Accordingly, 205 ADHD and ADHD-NOS case records were evaluated. The diagnosis of 205 patients' records and their comorbid psychiatric disorders was made clinically by the consensus of child psychiatry residents and the clinical supervisor according to DSM-IV-TR criteria (4). The diagnosis of ADHD was based on 6 of the 9 attention deficit criteria, 6 of the 9 hyperactivity criteria, onset of symptoms before the age of 6 years, the presence of symptoms in at least 2 different settings, symptoms having continued for at least 6 months and an impairment of functionality. The ADHDNOS was diagnosed for those patients who did not meet the criteria for ADHD (e.g. age, number of symptoms), but who had a behavioral pattern marked by sluggishness, daydreaming, and hypoactivity.

Inclusion criteria were a primary diagnosis of ADHD or ADHD-NOS according to DSM-IV-TR criteria, adequate information on laboratory values and psychometric measures in the patient records and application to the 
outpatient department during the specified time-frame. Patients with comorbid medical and psychiatric disorders were included. Both ADHD and ADHD-NOS groups received methylphenidate treatment at $1 \mathrm{mg} / \mathrm{kg} /$ day. Those patients with inadequate records were excluded. Ethics committee approval of the study was obtained from Bolu Abant Izzet Baysal University Clinical Trials Ethics Committee (date: 16.05.2018, number: 164).

\section{Measures}

Turgay DSM-IV-Based Child and Adolescent Behavior Disorders Screening and Rating scale (T-DSM-IV-S): This parent and Teacher-reported scale was developed by Turgay by transforming the DSM-IV criteria into questions without changing their meanings and includes 9 items for attention deficit, 6 items for hyperactivity, 3 items for impulsivity, 8 items for oppositional defiant disorder, and 15 items for conduct disorder. Each item is rated on a scale of $0=$ none, $1=$ occasional, $2=$ much, and $3=$ very much. When subscales are evaluated, 2 to 3 points per item are assessed as symptomatic (1), while 0 to 1 are assessed as nonsymptomatic (0) (26). The validity and reliability study of this scale was established previously (27).

The Screen for Anxiety Related Emotional Disorders (SCARED): There are 41 questions on this scale. It asks parents to show how often an explanatory sentence about how their children felt correct during the previous three months. Participants can choose from 0 to 2 points (28). When subscales are evaluated, 2 points for an item is assessed as symptomatic (1), while 0 to 1 are assessed as not symptomatic (0). Both the child's and parent's reports were used. The scale also includes generalized anxiety, separation anxiety, somatic/panic, social anxiety and school fear subscales. The SCARED Turkish form's validity and reliability were established by Cakmakci (29).

The Clinical Global Impression-Severity scale (CGI-S): $\mathrm{CGI}-\mathrm{S}$ is a scale that measures the functionality that varies with treatment evaluated by clinicians (30). The CGI-S score is a 7-point scale of disease severity. CGI-S is often used in Turkish Child and Adolescent psychiatry clinics. There are many Turkish studies using CGI-S. In this study, CGI-S was used to demonstrate symptom severity.

\section{Statistical Analysis}

For the data in this study, the Statistical Package for Social Sciences (SPSS) version 22.0 was used. Summary statistics are given for continuous variables. Unless otherwise stated, this refers to the number of patients (n), mean and standard deviation (SD). Categorical data are presented as absolute or relative frequencies. Demographic data were compared using the chi-square tests by applying the corrections of Yates and Fisher when necessary. The normal distribution of the data was examined with the Kolmogorov-Smirnov method. Since the distribution of the data was normal, group comparisons were assessed by Student t-test or One-Way ANOVA according to the group numbers. The relationship between continuous variables was made using pearson correlation analysis. All tests were considered statistically significant for $\mathrm{p}<0.05$.

\section{Results}

The records of 205 patients were analyzed. Of these, 99 (48.3\%) patients were ADHD and 106 (51.7\%) patients were ADHD-NOS. In the ADHD group, the average age of the children was $10.88 \pm 3.02$ years, while that of the children in the ADHD-NOS group was $9.93 \pm 2.49$ years. A significant difference was determined between the average ages of the groups $(p=0.015)$. Thirty-four children in the ADHD group were female, and 34 children in the ADHD-NOS group were female. There was no statistically significant difference between the groups in terms of gender $(p=0.73)$. There were 45 cases with a positive family history and 51 cases with a medical disease history in the ADHD group. In the ADHDNOS group, there were 54 cases with a positive family history and 48 cases with a medical disease history. Both family history and medical disease history between the two groups did not display statistically significant differences $(p=0.095, p=0.958$ respectively) (Table I). T-DSM-IV-

Table I. Comparison of sociodemographic data of the attention-deficit hyperactivity disorder not otherwise specified and attention deficit hyperactivity disorder groups

\begin{tabular}{|c|c|c|c|}
\hline & $\begin{array}{l}\text { ADHD-NOS } \\
\text { group } \\
(n=99)\end{array}$ & $\begin{array}{l}\text { ADHD } \\
\text { group } \\
(n=106)\end{array}$ & p \\
\hline Age & $10.88 \pm 3.02$ & $9.93 \pm 2.49$ & 0.015 \\
\hline \multicolumn{4}{|l|}{ Gender } \\
\hline Male & 65 & 72 & \multirow{2}{*}{0.73} \\
\hline Female & 34 & 34 & \\
\hline \multicolumn{4}{|c|}{ Family history } \\
\hline Positive & 54 & 45 & \multirow{2}{*}{0.095} \\
\hline Negative & 45 & 61 & \\
\hline \multicolumn{4}{|c|}{ Medical disease history } \\
\hline Positive & 48 & 51 & \multirow{2}{*}{0.958} \\
\hline Negative & 51 & 55 & \\
\hline
\end{tabular}

ADHD: Attention deficit hyperactivity disorder, ADHD-NOS: Attention-deficit hyperactivity disorder not otherwise specified, n: Number of patients 
S-parent subscales, SCARED subscales and CGI-S scores between the two groups are presented in Table II.

Comorbid psychiatric disorders were detected in 144 cases $(70.24 \%)$ of the 205 cases included in the study. It was found that conduct disorder (28.29\%) and specific learning difficulties (21.95\%) were the most common comorbid psychiatric disorders. While 71 of these 144 cases had only one comorbid psychiatric disorder, the other 73 cases had multiple comorbid psychiatric disorders. It was found that 76 cases in the ADHD group and 68 cases in the ADHD-NOS group had comorbid psychiatric disorders. There was no statistically significant difference between the groups in terms of comorbid psychiatric disorders $(p=0.531)$.

Iron and ferritin levels were measured in 81 of the 205 patients participating in the study. Hemoglobin levels were measured in 61 of the 205 patients participating in the study. While the averages of iron and ferritin levels were $65.53 \pm 33.21$ and $38.77 \pm 44.66$ respectively in the ADHD group $(n=47)$, they were $73.04 \pm 27.08$ and $32.83 \pm 17.11$ respectively in the ADHD-NOS group ( $n=34)$. No statistically significant difference was determined between the groups in terms of iron and ferritin levels ( $p>0.05)$. While the average of the hemoglobin level was $13.66 \pm 4.24$ in the ADHD group $(n=39)$, it was $12.77 \pm 1.06$ in the ADHD-NOS group $(n=22)$. There was no statistically significant difference between the groups in terms of hemoglobin levels ( $p=0.339)$ (Table III).
When the relationship between iron and ferritin levels and comorbidity was evaluated, iron and ferritin levels were $75.18 \pm 33.51$ and $33.56 \pm 16.39$ respectively in the noncomorbid group $(n=27)$, and they were $63.89 \pm 29.70$ and $33.91 \pm 20.82$ respectively in the single comorbid group $(n=24)$, and they were $65.81 \pm 29.48$ and $40.61 \pm 53.90$ respectively in the multiple comorbid group $(n=30)$. There was no statistically significant difference between the three groups in terms of iron and ferritin levels $(p=0.453, p=0.709$ respectively). When the relationship between hemoglobin level and comorbidity was evaluated, hemoglobin levels were $13.91 \pm 5.31$ in the non-comorbid group $(n=25)$, they were $12.90 \pm 1.04$ in the single comorbid group $(n=20)$, and they were $13.02 \pm 0.73$ in the multiple comorbid group $(n=16)$. There was no statistically significant difference between the three groups in terms of hemoglobin levels $(p=0.217)$. When the relationship between the total number of psychiatric diagnosis and iron, ferritin and hemoglobin levels was assessed, a statistically significant negative correlation was found between the total number of diagnosis and ferritin levels $(r=-351, p=0.001)$, but the same relationship with iron and hemoglobin levels was not observed ( $p>0.05)$.

When the relationship between the symptoms of ADHD and iron, ferritin and hemoglobin was evaluated, while there was a significant negative correlation between ADHD Hemolytic Anemia (HA) sub-scores and iron levels

Table II. Comparison of disorders screening and rating scale -parent subscales, the screen for anxiety related emotional disorders subscales and the clinical global impression-severity scale scores of the attention-deficit hyperactivity disorder not otherwise specified and attention deficit hyperactivity disorder groups

\begin{tabular}{|l|l|l|l|}
\hline \multicolumn{2}{|l|}{ ADHD-NOS group (n=99) } & ADHD group (n=106) & p \\
\hline T-DSM-IV-S-parent & $2.41 \pm 1.72$ & $6.28 \pm 2.15$ & $<0.001$ \\
\hline Attention & $1.40 \pm 1.85$ & $4.52 \pm 3.18$ & $<0.001$ \\
\hline Hyperactivity & $1.45 \pm 2.02$ & $3.56 \pm 2.99$ & $<0.001$ \\
\hline Opposition-defiance & $0.11 \pm 0.46$ & $0.56 \pm 1.22$ & 0.001 \\
\hline CD & $21.34 \pm 11.41$ & $42.37 \pm 17.02$ & $<0.001$ \\
\hline Total score & & & 0.441 \\
\hline SCARED & $1.85 \pm 2.31$ & $2.29 \pm 2.47$ & 0.001 \\
\hline Somatic/panic & $1.32 \pm 1.98$ & $3.11 \pm 2.64$ & 0.003 \\
\hline Generalized anxiety & $1.43 \pm 1.68$ & $2.68 \pm 1.92$ & 0.106 \\
\hline Separation anxiety & $2.09 \pm 1.95$ & $2.85 \pm 2.22$ & 0.050 \\
\hline Social anxiety & $0.53 \pm 1.31$ & $1.15 \pm 0.142$ & 0.007 \\
\hline School phobia & $25.89 \pm 12.86$ & $34.00 \pm 12.89$ & $<0.001$ \\
\hline Total score & $3.39 \pm 0.74$ & $4.55 \pm 0.64$ & \\
\hline CGI-S & & \\
\hline $\begin{array}{l}\text { ADHD: Attention deficit hyperactivity disorder, ADHD-NOS: Attention-deficit hyperactivity disorder not otherwise specified, SCARED: The screen for anxiety related } \\
\text { emotional disorders, CGI: The clinical global impression-severity scale, T-DSM-IV-S: Disorders screening and rating scale, n: Number of patients }\end{array}$ \\
\hline
\end{tabular}


$(p=0.027)$, and between ADHD AD sub-scores and ferritin ( $p=0.011)$, no correlation was found between the symptoms of ADHD and hemoglobin ( $p>0.05$ ) (Table IV). When the relationship between SCARED scores and iron, ferritin and hemoglobin levels was evaluated, no relationship was found between iron, ferritin and hemoglobin levels and the SCARED subscales and total scores ( $p>0.05)$.

Table III. Comparison of iron, ferritin and hemoglobin levels of the attention-deficit hyperactivity disorder not otherwise specified and attention deficit hyperactivity disorder groups

\begin{tabular}{|l|l|l|l|} 
& $\begin{array}{l}\text { ADHD-NOS } \\
\text { group }\end{array}$ & $\begin{array}{l}\text { ADHD } \\
\text { group }\end{array}$ & p \\
\hline $\begin{array}{l}\text { Iron } \mu \mathrm{g} / \mathrm{dL} \\
\text { (rr: } 60-180 \mu \mathrm{g} / \mathrm{dL})\end{array}$ & $73.04 \pm 27.08$ & $65.53 \pm 33.21$ & 0.349 \\
\hline $\begin{array}{l}\text { Ferritin, } \mathrm{ng} / \mathrm{mL} \\
\text { (rr: } 10-204 \mathrm{ng} / \mathrm{mL})\end{array}$ & $32.83 \pm 17.11$ & $38.77 \pm 44.66$ & 0.464 \\
\hline $\begin{array}{l}\text { Hemoglobin g/dL } \\
\text { (rr: } 11.5-17.5 \mathrm{~g} / \mathrm{dL})\end{array}$ & $12.77 \pm 1.06$ & $13.66 \pm 4.24$ & 0.339 \\
\hline
\end{tabular}

ADHD: Attention deficit hyperactivity disorder, ADHD-NOS: Attention-deficit hyperactivity disorder not otherwise specified, rr: Reference range

Table IV. Examination of the relationship between attention deficit hyperactivity disorder symptoms and iron, ferritin and hemoglobin levels (Pearson correlation analysis)

\begin{tabular}{|c|c|c|c|c|}
\hline & & $\begin{array}{l}\text { Iron } \\
\text { level }\end{array}$ & $\begin{array}{l}\text { Ferritin } \\
\text { level }\end{array}$ & $\begin{array}{l}\text { Hemoglobin } \\
\text { level }\end{array}$ \\
\hline \multicolumn{5}{|c|}{ T-DSM-IV-S-parent } \\
\hline Attention & $\begin{array}{l}r \\
p\end{array}$ & \begin{tabular}{|l}
-0.118 \\
0.398
\end{tabular} & $\begin{array}{l}-0.303 \\
0.011\end{array}$ & $\begin{array}{l}0.136 \\
0.350\end{array}$ \\
\hline Hyperactivity & r & \begin{tabular}{|l|}
-0.303 \\
0.027 \\
\end{tabular} & \begin{tabular}{|l}
-0.077 \\
0.527 \\
\end{tabular} & $\begin{array}{l}0.197 \\
0.175 \\
\end{array}$ \\
\hline Total score & r & $\begin{array}{l}-0.231 \\
0.095\end{array}$ & $\begin{array}{l}-0.127 \\
0.294\end{array}$ & $\begin{array}{l}0.186 \\
0.202\end{array}$ \\
\hline
\end{tabular}

\section{Discussion}

In this retrospective study, we aimed to compare the levels of iron, ferritin and hemoglobin in children with ADHD and ADHD-NOS, and to assess the relationship between ADHD symptom severity, anxiety symptom severity and iron, ferritin and hemoglobin levels. While we did not find significant differences between the ADHD and ADHDNOS groups in terms iron, ferritin and hemoglobin levels, we found a statistically significant negative correlation between ADHD HA symptom severity and iron levels, and ADHD AD symptom severity and ferritin levels. We also found a statistically significant negative correlation between the total number of psychiatric diagnoses and ferritin levels.
The main outcome of this study is that we have observed a significant negative correlation between ADHD HA symptom severity and iron levels, and ADHD AD symptom severity and ferritin levels. This means that as the iron level decreases, ADHD HA symptom severity increases, and as the ferritin level decreases, ADHD AD symptom severity increases. In studies evaluating iron, ferritin and ADHD relations, $A D H D$ and healthy control groups were compared for iron and ferritin levels (19,31-37). The data comparing ADHD and healthy control in terms of iron, ferritin was inconsistent. Some studies have shown no significant difference in ferritin levels between ADHD and healthy groups $(31,33)$, while others found significantly lower ferritin levels in children with ADHD (19,34-37). In a meta-analysis study, it was shown that serum ferritin levels were lower in ADHD patients than healthy controls. In the same meta-analysis, no relation was found between serum iron levels and ADHD (38). In studies evaluating the correlation between ADHD symptoms and ferritin levels, the data were inconsistent. While some studies had different findings from our study in that ADHD hyperactivity scores were negatively associated with serum ferritin level in ADHD (39-41), Menegassi et al. (32) found no relationship between ADHD symptoms and ferritin level.

Iron is a crucial element which plays a central role in brain processes (42). Therefore, ID causes structural and functional brain abnormalities such as dopamine metabolism alterations, energy metabolism and myelination (43). ID is associated with ADHD etiopathophysiology with several mechanisms (21). While there was a direct relationship between ADHD symptom and ferritin, the same direct relationship for $A D H D$ and iron level could not be found in the literature. By finding a negative correlation between ADHD HA symptom severity and iron level; we believe our study can contribute to the ADHD literature.

We also observed a significant negative correlation between the total number of psychiatric diagnosis and ferritin levels. This means that as the number of psychiatric diagnosis increases, the ferritin level decreases. Comorbidities are common in ADHD (9). In one study, serum ferritin levels were found to be higher in psychiatric disorders other than ADHD. (19). In a meta-analysis study, it was found that patients with ADHD as well as serum ferritin levels in other psychiatric comorbidity are higher than those without psychiatric comorbidity, which did not align with our findings. This difference in the literature and our study can be explained by different diet and pathophysiologic changes related to other psychiatric disorders. 
Another finding we have in our study is that there is no significant difference between the ADHD and ADHD-NOS groups with regard to iron, ferritin and hemoglobin levels. Our study may be a contribution to the literature on this subject. There is no study in the literature assessing the association between ADHD and ADHD-NOS in terms of iron, ferritin and hemoglobin levels.

\section{Study Limitations}

Our findings should be evaluated within the context of certain limitations. Firstly, this study did not have a healthy control group. Secondly, the study was retrospective and depended on information recorded routinely in clinical records. This dependence resulted in some missing data which may have affected the results. Thirdly, the study was conducted on a clinical sample evaluated at a single center and may not reflect patient populations in other centers or in the community.

Fourthly, the laboratory evaluations were conducted as part of the baseline examination prior to commencing pharmacotherapy at the study center but due to dependence on patient charts, we could not ascertain whether the patients were drug naive or were receiving treatment at the time of evaluations. Despite these limitations, this is the only study that we are aware of which evaluates psychometric features and laboratory values of children diagnosed with ADHD and ADHD-NOS.

\section{Conclusion}

In conclusion, a significant negative correlation between ADHD HA symptom severity and iron levels, and ADHD AD symptom severity and ferritin levels and an inverse relationship between the number of psychiatric diagnoses and ferritin with ADHD has been shown by our study. These findings suggest that iron and ferritin levels are related to ADHD symptoms and assessing ferritin levels may be beneficial if the number of psychiatric diagnoses increases in children with ADHD. However, there is a need for further study in this area because our findings and the literature available to date are both inadequate and inconsistent to generalize.

\section{Ethics}

Ethics Committee Approval: Ethics committee approval of the study was obtained from Bolu Abant Izzet Baysal University Clinical Trials Ethics Committee (date: 16.05.2018, number: 164).

Informed Consent: Retrospective study.

Peer-review: Externally peer-reviewed.

\section{Authorship Contributions}

Concept: Y.Ö., Z.T., Design: Y.Ö., N.D., Data Collection or Processing: Y.Ö., Z.T., N.D., Analysis or Interpretation: A.E.T., Literature Search: Z.T., N.D., Writing: Y.Ö., A.E.T.

Conflict of Interest: No conflict of interest was declared by the authors.

Financial Disclosure: The authors declared that this study received no financial support.

\section{References}

1. American Psychiatric Association. 'Diagnostic and statistical manual of mental disorders. 5th ed. Text Revision. Washington, DC: American Psychiatric Association Pres, 2013.

2. Biederman J, Faraone SV. Attention deficit hyperactivity disorder. Lancet 2005; 66:237-48.

3. Ersan EE, Dogan $\mathrm{O}$, Dogan $\mathrm{S}$, Sümer $\mathrm{H}$. The distribution of symptoms of attention-deficit/hyperactivity disorder and oppositional defiant disorder in school age children in Turkey. Eur Child Adolesc Psychiatry 2004; 13:354-61.

4. American Psychiatric Association. 'Diagnostic and statistical manual of mental disorders. 4th ed. Text Revision. Washington, DC: American Psychiatric Association Pres, 2000.

5. Faraone SV, Biederman I, Spencer T), et al. Diagnosing adult attention deficit hyperactivity disorder: Are late onset and subthreshold diagnoses valid? Am / Psychiatry 2006; 163:1720-29.

6. Biederman J. Attention-deficit/hyperactivity disorder: a selective overview. Biol Psychiatry 2005; 57:1215-20.

7. Faraone SV, Perlis RH, Doyle AE, et al. Molecular genetics of attention-deficit/hyperactivity disorder. Biol Psychiatry 2005; 57:1313-23.

8. Voeller KKS. Attention-deficit hyperactivity disorder (ADHD). I Child Neurol 2004; 19:798-814.

9. Thapar A, Cooper M. Attention deficit hyperactivity disorder. Lancet 2016; 387:1240-50.

10. Beard JL, Connor JR. Iron status and neural functioning. Annu Rev Nutr 2003; 23:41-58.

11. Beard J. Recent evidence from human and animal studies regarding iron status and infant development. I Nutr 2007; 137:524-30.

12. Yu GS, Steinkirchner TM, Rao GA, Larkin EC. Effect of prenatal iron deficiency on myelination in rat pups. Am I Pathol 1986; 125:620-4.

13. Connor JR, Menzies SL. Relationship of iron to oligodendrocytes and myelination. Glia 1996; 17:83-93.

14. de Andraca I, Castillo M, Walter T. Psychomotor development and behavior in iron-deficient anemic infants. Nutr Rev 1997; 55:125-32.

15. Youdim MB and Yehuda S. The neurochemical basis of cognitive deficits induced by brain iron deficiency: involvement of dopamine-opiate system. Send to Cell Mol Biol (Noisy-legrand) 2000; 46:491-500.

16. Rucklidge JJ, Johnstone J, Kaplan BJ. Nutrient supplementation approaches in the treatment of ADHD. Expert Rev Neurother 2009; 9:461-76. 
17. Pelsser LM, Frankena K, Toorman I, Savelkoul HF, Pereira RR, Buitelaar JK. A randomised controlled trial into the effects of food on ADHD. Eur Child Adolesc Psychiatry 2009; 18:12-9.

18. Sinn N. Nutritional and dietary influences on attention deficit hyperactivity disorder. Nutr Rev 2008; 66:558-68.

19. Cortese S, Azoulay R, Castellanos FX. Brain iron levels in attention-deficit/hyperactivity disorder: a pilot MRI study. World I Biol Psychiatry 2012; 13:223-31.

20. Sever $Y$, Ashkenazi A, Tyano S, Weizman A. Iron treatment in children with attention deficit hyperactivity disorder. A preliminary report. Neuropsychobiology 1997; 35:178-80.

21. Cortese $S$, Angriman $M$, Lecendreux $M$, Konofal $A$. Iron and attention deficit/hyperactivity disorder: What is the empirical evidence so far? A systematic review of the literature. Expert Rev Neurother 2012; 12:1227-40.

22. Lozoff $B$, De Andraca I, Castillo $M$ et al. Behavioral and developmental effects of preventing iron-deficiency anemia in healthy full-term infants. Pediatrics 2003; 112:846-54.

23. Bruner AB, Joffe A, Duggan AK, Casella JF, Brandt J. Randomised study of cognitive effects of iron supplementation in nonanaemic iron-deficient adolescent girls. Lancet 1996; 348:992-6.

24. Felt BT, Beard IL, Schallert T, et al. Persistent neurochemical and behavioral abnormalities in adulthood despite early iron supplementation for perinatal iron deficiency anemia in rats. Behav Brain Res 2006; 171:261-70.

25. Kim J, Wessling-Resnick M. Iron and mechanisms of emotional behavior. I Nutr Biochem 2014; 25:1101-07.

26. Turgay A. The DSM-IV Based child and adolescent behavior rating scale. Ontario, Integrative Therapy Institute, Canada, 1995. Unpublished data.

27. Ercan ES, Amado S, Somer O, Çikoğlu S. Development of a test battery for the assessment of Attention Deficit Hyperactivity Disorder. Turk J Chıld Adolesc Ment Health 2001; 8:132-44.

28. Birmaher B, Khetarpal S, Brent $D$, et al. The screen for child anxiety related emotional disorders (SCARED): Scale construction and psychometric characteristics. I Am Acad Child Adolesc Psychiatry 1997; 36:545-53.

29. Cakmakci FK. The reliability and validity study of the screen for child anxiety relatedemotional disorders (SCARED). Unpublished Expertise Thesis, Department of child and adolescent psychiatry, Kocaeli University, Faculty of Medicine, Kocaeli, 2004.

30. Guy W. Clinical global impression scale. The ECDEU assessment manual for psychopharmacology-revised. volume DHEW Publ No ADM 1976; 338:218-22.
31. Donfrancesco R, Parisi P, Vanacore N, Martines F, Sargentini V, Cortese $S$. Iron and ADHD: time to move beyond serum ferritin levels. / Atten Disord 2013; 17:347-57.

32. Menegassi M, Mello ED, Guimara es LR, et al. Food intake and serum levels of iron in children and adolescents with attentiondeficit/hyperactivity disorder. Revista Brasileira de Psiquiatria 2010; 32:132-8.

33. Kwon HJ, Lim MH, Ha M, et al. Transferrin in korean children with attention deficit hyperactivity disorder. Psychiatry Investig 2011; 8:366-71.

34. Konofal E, Lecendreux M, Arnulf I, Mouren MC. Iron deficiency in children with attention-deficit/hyperactivity disorder. Arch Pediatr Adolesc Med 2004; 158:1113-5.

35. Konofal E, Cortese S, Marchand M, Mouren MC, Arnulf I, Lecendreux $M$. Impact of restless legs syndrome and iron deficiency on attention-deficit/hyperactivity disorder in children. Sleep Med 2007; 8:711-5.

36. Juneja $M$, Jain $R$, Singh $V$, Mallika $V$. Iron deficiency in Indian children with attention deficit hyperactivity disorder. Indian Pediatr 2010; 47:955-8.

37. Mahmoud MM, El-Mazary AA, Maher RM, Saber MM. Zinc, ferritin, magnesium and copper in a group of Egyptian children with attention deficit hyperactivity disorder. Ital / Pediatr 2011; 37:60.

38. Wang $Y$, Huang $L$, Zhang $L$, et al. Iron status in Attention-Deficit/ Hyperacitivity Disorder: A systematic review and meta-analysis. PLoS One 2017;12.

39. Percinel I, Yazici KU, Ustundag B. Iron deficiency parameters in children and adolescents with Attention-Deficit/Hyperactivity Disorder. Child Psychiatry Hum Dev 2016; 47:259-69.

40. Oner P, Oner O, Azik FM, Cop E, Munir KM. Ferritin and hyperactivity ratings in attention deficit hyperactivity disorder. Pediatr Int 2012; 54:688-92.

41. Oner $\mathrm{O}$, Oner $\mathrm{P}$, Bozkurt $\mathrm{OH}$, et al. Effects of zinc and ferritin levels on parent and teacher reported symptom scores in attention deficit hyperactivity disorder. Child Psychiatry Hum Dev 2010; 41:441-7.

42. Lozoff B, Beard J, Connor J, Barbara F, Georgieff M, Schallert T. Long-lasting neural and behavioral effects of iron deficiency in infancy. Nutr Rev 2006; 64:34-43.

43. Georgieff MK. Long-term brain and behavioral consequences of early iron deficiency. Nutr Rev 2011; 69;43-8. 